\title{
Wie viel Syntax braucht die Semantik, und wie viel Semantik enthält die Syntax?
}

\author{
Hubert Haider \\ Fachbereich Linguistik, Universität Salzburg, Österreich
}

The nature of the syntax-semantics interface is a controversial issue in grammar theory. Current theorizing in Generative Grammar endorses a 'tight fit' conception. Abstract syntax is to provide distinct structural configurations for distinct semantic entities in terms of semantically typed functional heads that project functional phrases. Positive and convincing evidence for the tight fit approach is wanting. The opposite viewpoint, once called the autonomy viewpoint in Generative Grammar, takes syntax to be a module of grammar that is not necessarily tailored to the needs of semantics. It is an algorithm that maps strings on structures and vice versa. The semantic construction algorithm maps syntactic structure on adequate semantic domains. - In this contribution, evidence from three areas syntax of adverbials, syntax of negation, alleged semantic correlates of argument structure are reviewed in order to clarify which of the two viewpoints is more likely to be adequate. It is argued that the autonomy approach is superior.

\section{EINLEITUNG}

Die zwei Fragen im Titel konklusiv zu beantworten, hieße, das Syntax-Semantik-Verhältnis in der Grammatik endgültig expliziert zu haben. Dies lässt sich allerdings nicht theorieneutral bewerkstelligen, denn jedes Grammatiktheoriemodell beantwortet zumindest implizit und nicht immer vollständig - diese Fragen. Zumindest die polaren Standpunkte im Spektrum der Antworten lassen sich jedoch relativ leicht charakterisieren:

Einer der beiden Standpunkte ist der folgende: Die syntaktische Strukturierung ist eigengesetzlich gesteuert und unabhängig von der semantischen Repräsentation. Die Syntax liefert für die Semantikkonstruktion bloß die hierarchisch organisierten Strukturen samt Abhängigkeitsbeziehungen für die in einer linearen Abfolge organisierten terminalen Elemente. Die ,Syntax' ist die ,Oberflächensyntax'.

Der andere, entgegengesetzte Standpunkt ist: Die syntaktische Strukturierung ist letztlich homomorph mit der semantischen Struktur. Sie ist überdies so differenziert, dass sich eine direkte Abbildung von syntaktischen Strukturen auf semantische Repräsentationen erzielen lässt. Die ,Syntax' ist die Syntax der Semantik. Repräsentativ für diesen Ansatz ist das Modell der Generativen Grammatik.

Die LF-Syntax, wie sie sich in Arbeiten der Generativen Grammatik präsentiert 
(vgl. Hornstein 1995), betrachtet die Logische Form (= LF) als jene syntaktische Repräsentation eines sprachlichen Ausdrucks, die allen Anforderungen der semantischen Interpretation an hierarchischer und kategorialer Strukturierung genügt. Syntax und Semantik konvergieren in der LF-Struktur als einer syntaktischen Repräsentation. Folgendes vereinfachte Beispiel möge dies veranschaulichen:

1)
a. God knows everything
b. Für alle $\mathrm{x},[\mathrm{WISSEN}(\mathrm{g}, \mathrm{x})]$
c. $\left[\right.$ Everything $g_{\mathrm{i}}\left[\operatorname{god}\left[\right.\right.$ knows $\left.\mathrm{e}_{\mathrm{i}}\right]$

Die prädikatenlogische Repräsentation des Satzes (1a), wie sie in (1b) angedeutet ist, hat in der LF-Syntax eine direkte strukturelle Entsprechung. Die L (ogische) F(orm) von (1a) ist andeutungsweise in (1c) widergegeben: Quantifizierende Ausdrücke, wie das Objekt in (1a) werden in der semantischen Repräsentation auf Quantoren abgebildet, die eine Variable binden (1b). In der LF-Repräsentation positioniert eine syntaktische Operation ("quantifier raising") den quantifizierenden Ausdruck in die Position am Satzanfang. Die Ausgangsposition (, $\mathrm{e}_{\mathrm{i}}{ }^{\prime)}$ ) wird auf die Variable in der semantischen Repräsentation abgebildet, der angehobene Ausdruck auf den Quantor. Auf diese Weise wird bereits in der syntaktischen Repräsentation die pränexe Form für die semantische Repräsentation erzeugt. Die LF-Theorie geht davon aus, dass die relevante syntaktische Repräsentation die für die semantische Interpretation notwendigen strukturellen Distinktionen bereitstellt.

In diese Betrachtungsweise muss angenommen werden, dass es verborgene syntaktische Operationen geben muss, denn im Englischen wird ja das quantifizierte Objekt gerade nicht an den Satzanfang gerückt, wie man an der Abfolge der Elemente in (1a) erkennen kann. Es ist daher zu fragen, ob die Annahme einer LF-Struktur als syntaktischer Repräsentation gerechtfertigt ist, oder ob hier die Syntax unnötigerweise in das Revier der Semantik eindringt. Sie wäre gerechtfertigt, wenn sich die Eigenschaften der LF-Syntax unmittelbar mit den Eigenschaften sonstiger, nicht-verborgener syntaktischer Oparationen decken. Es ist aber mittlerweile deutlich geworden, dass es dafür keine stichfeste empirische Evidenz gibt.

\section{2) a. Man hat keinem erlaubt, jedes Zimmer zu jeder Zeit zu betreten \\ b. Jedes Zimmer ${ }_{\mathrm{i}}$ hat man keinem erlaubt, $\mathrm{e}_{\mathrm{i}}$ zu jeder Zeit zu betreten}

Die beiden Sätze sind semantisch nicht äquivalent. In Satz (2b) hat der Allquantor Skopus über das negierte Existential, in (2a) ist das nicht der Fall. Das zeigt, dass in der LF-Repräsentation eine Anhebung wie in (2b) nicht möglich ist, obwohl es eine syntaktisch zulässige Operation wäre, wie das Beispiel (2b) zeigt. Andererseits gibt es aber auch semantische Beziehungen, denen keine zulässige syntaktische Operation entspricht. Als Beispiel möge ein anderer Fall von angenommener Anhebung dienen:
a. Wer hat protestiert, als man wen verhaftete?
b. *Wen hat er protestiert als man $e_{\mathrm{i}}$ verhaftete? 
Die LF-Theorie nahm ihren Ausgang von der beabsichtigten Vereinheitlichung der Interrogativkonstruktionen. Es gibt Sprachen (z.B. slawische), in denen in einer Mehrfachfrage alle Frageausdrücke an den Satzanfang gerückt werden. Andere Sprachen hingegen erlauben in Mehrfachfragen nicht, dass mehr als ein Frageelement vorangestellt wird. Dies gilt beispielsweise für alle germanischen Sprachen. Die Vereinheitlichung wurde darin gesehen, dass auf LF alle Elemente umgestellt werden. Nun zeigt aber (3b), dass eine syntaktische Umstellung blockiert ist, wenn dabei eine Adverbialsatzgrenze überschritten werden müsste. Für die LF-basierte Umstellung in (3b) müsste aber gerade diese universelle Restriktion aufgehoben werden. Die LF-Syntax kann folglich nicht die verborgene Fortsetzung genau der syntaktischen Operationen sein, die im ,sichtbaren' Bereich operieren. Damit verliert sie aber ihre Plausibilität.

Dieser Beitrag setzt sich das moderate Ziel, anhand von drei Phänomenbereichen eine Abschätzung vorzunehmen, in der Nähe von welchem der beiden Pole die adäquaten Antworten am Ehesten zu suchen und zu finden sein mögen. Die drei Phänomenbereiche sind i) die Adverbialsyntax, ii) die Syntax der Negation, und iii) die Argumentstruktur.

Jedes der angesprochenen Phänomene ist eines mit deutlichen Schnittstelleneigenschaften. Die Distribution der Adverbien korreliert mit ihrem semantischen Typ. Die offensichtliche Frage ist daher, ob die syntaktischen Eigenschaften sich direkt aus den semantischen ableiten lassen. Negation ist ein semantisches Konzept. Die syntaktische Realisierung sollte daher hinreichend Einblicke in den zur Debatte stehenden Fragenkomplex bieten. Argumentstruktur schließlich bezieht die zweite Semantikschnittstelle ein, nämlich die im Lexikon. Wieder ist die Frage, ob sich die Argumentstrukturtypen (in Sonderheit die Unterscheidung zwischen unakkusativischen und intransitiven Verben) als semantische Typen fassen lassen, oder ob es sich letztlich um eine formale Unterscheidung handelt, die semantisch befrachtet ist.

Es sei schon vorausgeschickt, dass es der erste der beiden genannten Standpunkte ist, der sich im Lichte der folgenden Auseinandersetzungen als der dem Verfasser plausibler erscheinende und daher von ihm favorisierte erweisen wird. Der Beitrag ist wie folgt gegliedert: Im folgenden Abschnitt wird kurz die Funktion der Syntaxkomponente im System der Grammatik umrissen. Daran schließen sich die bereits genannten Abschnitte über die drei Phänomenbereiche. Im letzten Abschnitt wird eine zusammenfassende Einschätzung angeboten.

\section{WozU SyntAX?}

Die Syntax einer natürlichen Sprache ist ein Algorithmus für die Konversion von Datenformaten. Dieser Algorithmus leistet eine Schnittstellenfunktion, indem er ansonsten inkongruente Datenstrukturen aufeinander abzubilden erlaubt. Im Falle der Lautsprache sind das einerseits die linear organisierte phonetisch-phonologische Struktur, und andererseits die als Funktor-Argument-Struktur rekonstruierbare semantische Repräsentation. Die syntaktische Struktur ist eine hierarchisch gegliederte ,Schachtel-in-Schachtel'-Struktur mit Abhängigkeitsbeziehungen. 
Die semantische Repräsentation einer Äußerung legt nicht nur die Wahrheitsbedingungen fest (= Semantik im engeren Sinn), sondern auch die kontextabhängigen pragmatischen Eigenschaften, die uns für Inferenzen aufgrund der Informationsstrukturierung zur Verfügung stehen, wie zum Beispiel die Fokus-Hintergrund Gliederung einer Äußerung.

Aus den Blickwinkel der Rezeption betrachtet, wird über der linearen Struktur die syntaktische Struktur entfaltet, die als Basis für die Semantikkonstruktion fungiert. Im umgekehrten Fall, also im Fall der Produktion, wird die syntaktische Struktur auf eine Linearisierung abgebildet. Die Syntax gewährleistet somit die rasche und effektive Komprimierung oder Entfaltung einer speziellen Struktur, die als Ein-/Ausgabe für die semantischen und phonologischen Repräsentationen dient und damit die Abbildung der beiden Repräsentationsebenen aufeinander gewährleistet.

Die Tatsache, dass Sprachen sich in ihren Linearisierungseigenschaften unterscheiden, nicht aber in ihren semantischen Bedingungen, ist ein erster Beleg dafür, dass die Semantik kein Diktat über die syntaktischen Strukturen ausübt. Es ist eher umgekehrt. Die Semantik ist auf die jeweils gegebenen syntaktischen Verhältnissen angewiesen, was in manchen Fällen dazu führt, dass eine Ausdrucksweise, die in einer Sprache möglich ist, in einer anderen nicht zur Verfügung steht.

Betrachten wir dazu ein einfaches Beispiel:

a. Who left when/*why? - He asked who left when/*why

b. Wer lief wann/weshalb weg? - Er fragte, wer wann/weshalb weggelaufen sei

In einer Sprache wie Englisch, stellvertretend für OV Sprachen ${ }^{1}$ wie z.B. auch Dänisch, Norwegisch, Schwedisch oder romanische Sprachen, lässt sich eine Mehrfachfrage, die das Subjekt und gleichzeitig den Grund oder die Art und We is e befragt, nicht in der Weise stellen, wie das im Deutschen, stellvertretend für OV Sprachen wie z.B. Niederländisch, Afrikaans, oder Japanisch möglich ist, nämlich als Mehrfachfrage. Dieser merkwürdige Unterschied ist keine bizarre Ausnahme sondern systematisch für VO Sprachen.

Der Grund liegt im Syntax-Semantik-Verhältnis. VO Sprachen stellen die für die semantische Wohlgeformtheit erforderliche syntaktische Konfiguration nicht zur Verfügung. Im speziellen Fall ist das eine Konstellation, in der ein Adverbial wie why (als adverbialer Frageoperator eines höheren semantischen Typs) syntaktisch so platziert sein muss, dass es als Operator über die jeweils erforderliche semantische Domäne fungieren kann. Im Falle von why ist das die Domäne, die das finite Verb enthält, denn why ist ein Operator der über propositionale Typen quantifiziert, nämlich die Menge der möglichen Gründe. In VO Sprachen gibt es aber keine mögliche syntaktische Position mit der geforderten Domäneneigenschaft, außer der Spitzenposition, wie in (5a). Doch auch diese Anordnung scheidet aus; diesmal allerdings aus einem Grund, der den zweiten der beteiligten Frageausdrücke betrifft, also das Subjekt in Form eines Frageausdruckes. 


$$
\begin{gathered}
\text { a. }{ }^{*} \text { Why } /{ }^{*} \text { When did WHO leave? - He asked *why/*when WHO left } \\
\text { b. Wann/Weshalb lief WER weg? - Er fragte, wann/we shalb WER } \\
\text { weggelaufen sei }
\end{gathered}
$$

Wiederum ist es die Syntax, die für den Unterschied verantwortlich ist: Weil in einer VO Sprache das Subjekt stets in einer speziellen Position zu stehen kommt, die nicht seine Grundposition als Argument des Verbums ist, trifft für ein VO Subjekt stets eine Bedingung zu, die in eine OV Sprache, in der das Subjekt ja in seiner Grundposition als Argument verharren darf, nicht greift. Diese Bedingung hat zur Folge, dass ein Interrogativ-Element in einer Spezifikatorposition obligatorisch als Operator fungiert, und in VO-Sprachen steht das Subjekt obligatorisch in einer Spec-Position. Um seine erforderliche Operatorfunktion bekleiden zu können, müsste das Subjekt der Interrogativausdruck sein, der den Satzmodus als Fragesatz determiniert oder es müsste zumindest eine Variable binden. Die erste Bedingung ist nicht erfüllbar, weil ja der adverbiale Frageausdruck vorangestellt wurde und damit zum Element wurde, das den Satzmodus bestimmt. Die zweite Bedingung ist in (5a) auch nicht erfüllt und somit bleibt ein Makel. (6) ist ein Beispiel für die Erfüllung dieser Bedingung. Das Subjekt in Form eines Interrogativausdruckes in seiner Oberflächenposition bindet entweder ein abhängiges Interrogativelement oder ein abhängiges Pronomen. In beiden Fällen ist somit die für ein Operatorenelement erforderliche Bindungseigenschaft gegeben.

Der anhand von (5) erörterte Makel kommt dadurch zustande, dass die Semantik eine Konstellation erforderlich macht, die Syntax diese aber nicht in jedem Fall bereithält. In OV-Sprachen ist die Konstellation verfügbar, in VO-Sprachen aber nicht.

\section{6) (?)Why did who $o^{\mathrm{i}}$ leave whom / hi is ${ }^{\mathrm{i}}$ partner}

Reflektieren wir nun kurz den beschriebenen Sachverhalt: Die Syntax bestimmt strukturelle Konstellationen, die in manchen Fällen es nicht erlauben, den dafür zutreffenden semantischen Erfordernissen gerecht zu werden. Die entsprechenden Äußerungen sind somit deviant. Die Tatsache, dass es abhängig von syntaktischen Konstellationen, die von Sprachtyp zu Sprachtyp unterschiedlich sein können, zu semantisch basierten Restriktionen kommt, ist Indiz dafür, dass es die Semantik ist, die auf bestimmte strukturelle Verhältnisse angewiesen ist. Stellt die Syntax diese nicht zur Verfügung, dann kann das die Semantik das nicht erzwingen.

Wäre die Syntax ein formales Grundgerüst, das seine Form und Funktion wesentlich der Semantik schuldet, sollte der eben diskutierte Fall gar nicht existieren können. Mit anderen Worten, Sprachen sollten sich nicht darin unterscheiden, dass in der einen Sprache ein Konstruktionstyp existiert (z.B. Mehrfachfragen), dessen Äquivalent in der anderen Sprache wegen Nicht-Erfüllbarkeit der strukturellen Bedingungen semantisch ausgeschlossen wird. Wenn die Semantik diktieren könnte, welche Strukturen die Syntax bereitzustellen habe, würde sie, da sich Sprachen in ihren rein semantischen 
Bedingungen kaum unterscheiden, in jeder Sprache die Strukturen vorfinden, die sie braucht. Wenn hingegen die Syntax eine eigengesetzlich bestimmte Subdomäne der Grammatik ist, wird die Semantik sich den syntaktischen Gegebenheiten fügen müssen, was - wie bereits gezeigt - dazu führen kann, dass sich Sprachen darin unterscheiden, welchen strukturellen Möglichkeitsraum sie der Semantik zur Verfügung stellen.

Dieser Gedanke - Sprachen variieren syntaktisch stark, semantisch aber kaum, was auf ein einseitiges Abhängigkeitsverhältnis zwischen Syntax und Semantik hindeutet - wird den roten Faden für die folgende Erörterung der drei Phänomenbereiche bilden.

\section{Adverbialsyntax}

Es ist eine bekannte Tatsache, dass die adverbialen Elemente abhängig von ihrem semantischen Typ über die Sprachen hinweg in einer stark präferierten bis mandatorisch festgelegten Abfolge auftreten. Hier ist ein Beispiel:
a. ${ }^{*}$ He has completely never solved the problem
b. *Er hat vollständig nie das Problem gelöst
c. *He has not completely [solved the problem unfortunately]
d. Vollständig ${ }_{\mathrm{i}}$ hat er [das Problem le ider $\mathrm{e}_{\mathrm{i}}$ nicht gelöst]

Ein Frequenzadverbial geht in der Grundabfolge einem Art\&Weise-Adverbial voraus (7a, b), wobei die Position an der Satzspitze in (7d) eine abgeleitete Position ist. Das Reihenfolgeverhältnis zwischen den beiden Adverbialkategorien ergibt sich letztlich aus dem jeweiligen semantischen Typ. Ein Frequenzadverb quantifiziert über Ereignisse; das Art\&Weise-Adverb spezifiziert den durch das Verb denotierten Prozess. Frequenz-Quantifikation schließt die Art-\&Weise-Modifikation als Eigenschaft eines Ereignisprädikats daher in seine Domäne ein. Die Konsequenz ist, dass ein Frequenzadverb strukturell einem Art\&Weise-Adverb übergeordnet ist.

Für den Kontrast zwischen (7c) und (7d) ist wieder der OV- versus VO-Charakter verantwortlich. Warum darf ein Satzadverb einem Objekt in einer OV-Sprache hinterdreingehen, nicht aber in einer VO-Sprache? Ein Satzadverb bringt eine p r o p ositionale Einstellung zum Ausdruck. Eine Proposition ist, nach Davidson (1980), eine Prädikation über eine Ereignisvariable. Diese Variable wird überdies zeitlich situiert. Das Satzadverb muss daher das finite Verb in seiner Domäne haben, denn dieses ist der Träger der Tempusmerkmale, deren semantische Interpretation die zeitliche Situierung der Ereignisvariablen steuern. In VO-Sprachen werden die Tempusmerkmale über eine funktionale Position verwaltet, die der VP vorausgehen. Es ist dies die Position, die im Englischen das finite Auxiliar im Deklarativsatz einnimmt. Ein Satzadverb genügt dem semantisch determinierten Domänenerfordernis nur dann, wenn es der VP vorangeht. Nur dann befindet sich das finite Verb selbst oder dessen Grundposition in der VP in der strukturellen Domäne des Adverbs:

8) a. He could $\mathrm{i}_{\mathrm{i}}\left[\mathrm{unfortunately}\left[\right.\right.$ not $\left[_{\mathrm{VP}-\mathrm{can}} \mathrm{e}_{\mathrm{i}}\left[_{\mathrm{VP}-\text {-solve }}\right.\right.$ solve the problem $\left.]\right]$

b. It is unfortunate that he could not solve the problem 
Das Faktum, dass sich auch die modale Modifikation, ausgedrückt mittels Modalverb, semantisch im Skopus des Satzadverb befindet (vgl. die Paraphrase in 8b), folgt daraus, dass sich die Grundposition des Modalverbs, angedeutet durch die koindizierte Leerstelle in (8a), in der Domäne des Satzadverbs befindet.

In VO-Sprachen hingegen werden die Finitmerkmale direkt am Verb in der VP verwaltet. Ein VP-internes Satzadverb hat somit das finite Verb und damit das Element, das die Ereignisvariable situiert, in seiner Domäne, und erfüllt damit die semantischen Anforderungen für ein Satzadverb. Gleiches gilt übrigens für Satzadverbien in adnominalen Attributen, wie in (9). Hier ist in beiden Fällen, Englisch wie Deutsch, der Kopf des Attributs das Element, das für die propositionale Situierung verantwortliche Element.

(9) a. das [leider völlig unverständliche] Papier

b. the [unfortunately completely incomprehensible] paper

Die Beispiele in (10) demonstrieren, dass ein Adverb, das eine propositionale Einstellung ausdrückt, tatsächlich eine Proposition fordert, denn in (10c) kann sich das Satzadverb nicht so, wie in (10a, b) auf das Prädikat roh beziehen. Mit anderen Worten, (10c) lässt sich nicht wie in (11a) paraphrasieren, sondern nur wie in (11b). Das sekundäre Prädikat roh in (10c) e t a bl i e r t keine Proposition, sondern bildet, zusammen mit dem Verb, ein komplexes Prädikat ,roh essen'.2

10) a. Das Ei ist leider noch roh

b. das leider noch rohe $\mathrm{Ei}$

c. Er aß das Ei leider roh

11) a. Er hat das Ei gegessen und es war leider noch roh

b. Es war leider so, dass er das Ei roh gegessen hat

Wenden wir uns nun den eingangs vorgestellten polaren Zugängen zu. Ein semantiknahes Modell vertritt Cinque (1999). Seiner Ansicht nach stellt die Syntax für jedes Adverbial eine funktionale Kopfposition zur Verfügung, deren Spezifikatorposition das jeweilige Adverb beherbergt. Die Gegenposition, der sich auch der Autor anschließt, vertritt Ernst (2002): Die Syntax bestimmt lediglich die möglichen Positionen für Adverbiale im Satz. Die Semantik entscheidet über die Interpretation als Funktion der Interpretation der jeweils unterschiedlichen strukturellen Domänen. So gibt es beispielsweise in VO-Sprachen weniger ,Adverbialnischen' als in OV-Sprachen, da einerseits die VP kompa k ${ }^{3}$ ist (12a vs. 12b), und da andererseits die Adjunktion von Adverbien an kopf-initiale Strukturen, wie z.B. an die VP, der Einschränkung unterliegt, dass der Kopf der adjungierten Phrase und das Adjunktionsziel adjazent sind (12c vs. 12d). Englisch ist dabei repräsentativ für eine VO-Sprache und Deutsch für eine OVSprache.

12) a. *He has solved completely some problems

b. Er hat irgendwelche Probleme vollständig gelöst 


\section{c. He has ((much) more) cleverly (*than I) handled the matter \\ d. Er hat die Sache sehr viel klüger (als ich) gehandhabt}

Die Annahme, dass Adverbiale in satzinterner Position die Spec-Position der jeweiligen abstrakten, funktionalen Adverb-Köpfe besetzen, macht eine klare und falsche Prognose, und scheidet damit als empirisch adäquate Modellierung aus. Die Prognose lautet: Wird ein eingebetteter Objektsatz vor eines der fraglichen Adverbiale gesetzt, befindet sich der Satz in einer abgeleiteten Position außerhalb der VP. Er ist entweder an eine funktionale Phrase adjungiert oder befindet sich in der Spec-Position eines funktionalen Kopfes. In beiden Fällen ist die Konsequenz dieselbe: Extraktion aus diesem Satz ist blockiert. Dass dies eine falsche Prognose ist, zeigt das Beispiel (13).

a. Wen $n_{\mathrm{i}}$ ist [damit $\mathrm{e}_{\mathrm{i}}$ überzeugen] leider auch ihm nicht gelungen?

b. Wen $n_{\mathrm{i}}$ hat [damit $\mathrm{e}_{\mathrm{i}}$ überzeugen] leider auch er erfolglos versucht?

Überdies schlägt negativ zu Buche, dass die Annahme einer syntaktisch geordneten Kaskade von funktionalen Phrasen für Adverbiale ein- und dasselbe Ordnungsprinzip doppelt kodiert, denn es ist die Semantik, die die relative Abfolge steuert. Es ist redundant, dasselbe Abfolgeverhältnis auch noch in der syntaktischen Strukturierung festzuschreiben. Dass es semantische Eigenschaften sind, und nicht strukturelle, die die relativen Einbettungsdomänen determinieren, erkenn man unschwer im Vergleich der Abfolgeverhältnisse von Adverbialen und von adnominalen Attributen. In beiden Fällen sind die Abfolgen semantisch und auf gleiche Weise gesteuert: Art\&WeiseModifikation ist in kopf-nächster Position; darüber kommt die Frequenzangabe, darüber die zeitbezogenen Angaben.

14) a. diegestrigen rechtzeitigen mehrfachen gründlichen Durchsuchungen der Räume

b. die [gestern rechtzeitig mehrfach gründlich durchsuchten] Räume

c. Man hat die Räume gestern rechtzeitig mehrfach gründlich durchsucht

Als unterstützendes Indiz für seine Annahme verweist Cinque auch darauf, dass in manchen Sprachen adverbiale Funktionen mittels verbaler Affixe kodiert werden und dass diese Affixe in spiegelbildlicher Abfolge zu der Abfolge der phrasale Adverbiale auftreten in den uns vertrauten Sprachen auftreten. Das erkläre sich daraus, dass das Verb sich von Kopf zu Kopf durch die funktionale Kaskade bewege und dabei die adverbialen Merkmale ablese, die morphologisch als Affixe ausbuchstabiert würden.

Dem sei entgegengehalten, dass die Spiegelbildabfolge der Affixe kein zwingendes Argument begründet. Sie lässt sich unschwer als Wortstruktureffekt erklären, und zwar in derselben Weise, wie für die phrasalen Adverbiale, nämlich über D o m ä n e n i n k l u s i o n bei der kompositionalen Konstruktion der Semantik eines Ausdruckes:

$$
\left[\left[\left[\text { Verb] Affix }{ }_{1}\right] \text { Affix }_{2}\right]\right.
$$

Wenn die beiden Affixe in (15) adverbiale Funktionen kodieren, so muss Affix $_{2}$ eine 


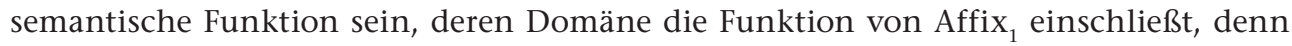
der kompositionale Aufbau der Wortstruktur steuert die kompositionale Konstruktion der semantischen Repräsentation und legt damit die relativen Einbettungsdomänen strukturell fest. Es ist daher nicht verwunderlich, dass die kompositionale Semantik in jedem Fall, sei es im Falls der Phrasenstruktur oder der Wortstruktur, die strukturellen Verhältnisse in derselben Weise respektiert. Daraus aber ergibt sich unmittelbar, dass die Abfolgen spiegelbildlich sein müssen, denn die Wortstruktur im Fall von (15) ist linksrekursiv, während die Phrasenstruktur rechts-rekursiv ist. Das aber ist eine syntaktische, und keine semantische Gegebenheit.

\section{Negation}

In vielen Sprachen wird Negation mittels einer Negationspartikel kodiert. Kontrovers diskutiert wird, ob diese Partikel als spezieller funktionaler Kopf zu betrachten sei, der in der syntaktischen Struktur eine Negationsphrase aufspanne, oder ob es bloß eine Partikel mit spezieller semantischer Funktion sei, die im Übrigen die Syntax einer Partikel hat. ${ }^{4}$ Die Frage ist, ob die Syntax eine syntaktische Kategorie ,Neg$\mathrm{P}^{\prime}$ vorzusehen habe, oder ob Negation bloß eine semantische Kategorie ist, die syntaktisch von einer Partikel repräsentiert wird.

Seit Pollock (1989) wird im Rahmen der Generativen Grammatik die Negationspartikel als Bestandteil einer Negationsphrase betrachtet, die ein Negationselement als funktionaler Kopf aufspannt. Damit wird auch eine Spec-Position eröffnet. Haegeman (1995: 107) erhebt es zum Prinzip, dass ein Negationsoperator in einer Spec-Kopf Konstellation zu einem NEG-Kopf stehen muss, und vice versa (,Neg-Kriterium'). Demnach sei mit der Satznegation stets eine Negationsphrase verbunden. Dieses Prinzip wird als Spezialfall einer allgemeinen Bedingung gesehen (cf. Haegeman 1995: 93, "affect-criterion"), wonach Operatorenmerkmale, wie z.B. Interrogativ- oder Fokusmerkmale, stets in einer Spec-Kopf-Konstellation grammatisch verwaltet würden. Das Haegemansche NegKriterium ist analog zum Wh-Kriterium, das Rizzi (1990) postulierte, konzipiert.

$$
\left[\ldots\left[\mathrm{NEG}-\mathrm{OP}\left[\mathrm{N}^{\circ}[\ldots]\right]\right]\right]
$$

In dem in (16) angegebenen Strukturschema (vgl. Hornstein 1995:168; Haegeman 1995: 115), kann, je nach Sprache, die Kopf- oder die Operatorbesetzung phonetisch stumm sein. Als Beispiel für die Präsenz beider Elemente dient das Französische. Der NegationsKopf ne ist ein Klitikum und tritt in der syntaktischen Struktur infolge Klitisierung am Verb auf, das der Träger der funktionalen Merkmale im Satz ist. Das zweite Element, nämlich pas, nimmt die Spezifikatorposition der NEG-P ein.

17) Je ne suis $_{\mathrm{i}}\left[_{\mathrm{Neg}-\mathrm{P}}\right.$ pas $\left[\mathrm{e}_{\mathrm{i}}\left[_{\mathrm{VP}} \mathrm{e}_{\mathrm{j}}[\right.\right.$ venue $\left.\left.\left.]\right]\right]\right]$

Für Deutsch und Niederländisch nimmt Haegeman an, dass die Negationspartikel ein Element in der Spezifikatorposition sei, und nicht der Kopf der Neg-Phrase. Dazu bewegen sie ausschließlich innertheoretische Erwägungen: Wäre die Negationspartikel 
der funktionale NEG-Kopf, würde er mit der Versetzung des finiten Verbs in V1- und V2-Sätzen interagieren und diese blockieren. Das finite Verb müsste sonst, da Köpfe bei Verschiebung keine Kopfpositionen überspringen können, an den Neg-Kopf klitisieren. Doch auch die Annahme, dass die Negationspartikel in Spec-Neg auftritt, ist problematisch. In diesem Fall sollte sie auch vorfeldfähig sein, was im Deutschen generell nicht der Fall ist (wohl aber in skandinavischen Sprachen).

\section{8)}

*Nicht kennt man im Deutschen diese Voranstellung

Wenden wir uns nun den zu erklärenden Fakten zu. Wenn es im Deutschen eine NEGPhrase gibt, muss sie die VP einschließen und damit vor der Grundposition des Subjekts und der Objekte stehen. Die Negationspartikel tritt im Deutschen allerdings zu verbnah auf, als dass die angenommene Grundkonstellation von Neg-P und VP dies zuließe.

a. dass hier wer was nicht begreift

b. *dass hier nicht wer was begreift

Die Abfolgen, die zu erwarten wären, wenn die Negationsphrase die VP dominiert, sind bei Satznegation ungrammatisch (19b). Faktum ist nämlich, dass indefinite Nominalausdrücke nicht im Skopus der Satznegation auftreten können, sondern durch negierte Indefinitausdrücke ersetzt werden müssen. Man müsste, um (19a) ableiten zu können, für diese Fälle annehmen und nachweisen, dass diese Ausdrücke durch obligatorisches Scramblings vorangestellt werden. Doch gerade die in (19) vorkommenden indefiniten $W$-Ausdrücke erweisen sich in den Standardfällen von Scrambling als umstellungsresistent.

20) a. ??dass hier was einer nicht begreift

b. dass hier einer was nicht begreift

Scrambling ist auch aus einem weiteren Grund eine ungünstige Lösung, denn die durch Scrambling bewirkte und zu erwartende Skopusambiguität ist bei Satznegation nicht nachzuweisen. In (21a) gibt es die sekundäre Lesart, die der Grundabfolge entspricht, nämlich (22a). Die Lesart (22b), die zu erwarten wäre, wenn in (21b) das Objekt über die Negation umgestellt worden wäre, ist aber nicht verfügbar.

21) a. Er hat genau zwei Bilder ${ }_{\mathrm{i}}$ niemandem $\mathrm{e}_{\mathrm{i}}$ gezeigt

b. Er hat genau zwei Bilder nicht verzollt

22) a. Es gibt niemandem, dem er genau zwei Bilder gezeigt hat

b. Es ist nicht der Fall, dass es genau zwei Bilder waren, die er verzollte

Eine Abfolge, die unter dem Neg-Kriterium zu erwarten wäre, wenn Scrambling nicht als obligatorisch postuliert wird, nämlich die Spitzenstellung der Negation wie in (23ac), findet man zwar im Deutschen, aber nur in Konditionalsätzen und in Objektsätzen in Form von indirekten Fragesätzen, nicht jedoch in deklarativen Komplementsätzen. 
Diese Stellung der Negationspartikel als Satznegation an der Spitze des Mittelfeldes ist in Deklarativsätzen und in Subjektsätzen, auch wenn es indirekte Fragesätze sind, unzulässig (vgl. 23d, e):

23) a. Wenn damals nicht die Polizei den Weg versperrt hätte, ...

b. Hätte damals nicht die Polizei den Weg versperrt, ...

c. Ich frage mich, ob nicht die Polizei den Weg versperren sollte

d. $\left(^{*}\right)$ Ich vermute, dass nicht die Polizei den Weg versperren wird

e. $\left(^{*}\right) \mathrm{Ob}$ nicht die Polizei den Weg versperrt hat, interessiert mich sehr

(23d, e) ist nur zulässig, wenn die Negation als Konstituentennegation der NP fungiert, nicht aber als Satznegation. Es wäre verfehlt, den Unterschied zwischen (23a-c) und (23d, e) auf einen Unterschied im Scrambling-Verhalten zurückführen zu wollen, treten doch in jedem Satz dieselben Mittelfeldelemente auf. Es ist die Stellung der Negationspartikel, die es zu charakterisieren gilt.

Auffällig ist auch, dass bei Spitzenstellung in indirekten Frage- und Konditionalsätzen die in den anderen Fällen zu beobachtende Unverträglichkeit von Negation mit Indefinitpronomina im Skopus der Negation nicht auftritt:

24) a. wenn dann nicht noch wer/einer/Max im letzten Augenblick einen Fehler macht

b. wenn dann nicht noch jeder/Max wem was zuflüstert

Bemerkenswert ist auch das unterschiedliche Verhalten bei anaphorischem Bezug, wenn sich das Antezedens des Pronomens im Skopus der Negation befindet (25b).

25) a. Wenn die nächste Zahl nicht gerade ist, muss sie durch 3 teilbar sein

b. Wenn nicht die nächste Zahli gerade ist, muss sie*i durch 3 teilbar sein

Dieser Effekt ist zu erwarten, wenn man einen Konstruktionsalgorithmus für die semantische Repräsentation wie in der DRT (cf. Kamp/Reyle 1993: 155) ansetzt: Das Antezedens im Konditionalsatz ist für das Pronomen im Hauptsatz unzugänglich, wenn es sich im Skopus der Negation befindet, da es sich dann in einer sub-DRS befindet.

Gibt es zwingende empirische Evidenz für die Annahme einer Neg-Phrase in der Satzstruktur des Deutschen? Haegeman (1995: 166-169) beruft sich auf einen Vorschlag von C. Hamann, wonach das Stellungsverhalten von AP-internen PPs mit einem negiertindefiniten Nominalausdruck direkt die Erwartungen bestätige.
a. weil Peter mit nichts zufrieden ist
[ambig]
b. weil Peter zufrieden mit nichts ist
[keine Satznegation]
c. weil Peter zufrieden ist mit nichts
[keine Satznegation]

Nur in der vorangestellten Version (26a) befinde sich der Negationsausdruck in der Spec-Position, oder in einer Position, von wo aus er diese c-kommandiere. Das sei der Grund, weshalb nur in (26a) der negierte Indefinitausdruck eine Satznegation bewirke. 
Diese Analyse ist allerdings nicht zwingend. Es reicht die Annahme, dass Satznegation nur dann vorliegen kann, wenn der Negationsausdruck den funktionalen Kopf, der die Ereignisvariable verwaltet, also das finite Verb im finiten Satz, oder das nicht-finite Matrixverb im satzwertigen Infinitiv c-kommandiert. ${ }^{6}$ Dies ist die generelle strukturelle Voraussetzung für skopussensitive Elemente. In (26a) ist diese Voraussetzung erfüllt, nicht aber in (26b, c). Der Grund ergibt sich aus dem syntaktischen Kohärenzverhalten. ${ }^{7}$ In (27) sind die relevanten Konstituentenstrukturen für (16) angegeben.
a. weil Peter mit nichts [zufrieden ist]
b. weil Peter [zufrieden [mit nichts]] ist
c. weil Peter [[zufrieden ist] [mit nichts]]

In unmittelbare Schwierigkeiten gerät der Haegemansche Vorschlag auch mit den Lizenzierungsbedingungen für negative Polaritätsausdrücke. Im Englischen ist der Determinator any ein Polaritätsausdruck. Er tritt anstelle von some im c-KommandoBereich von Negation auf.

28) a. I did not give anyone anything

b. I gave noone anything

c. *I gave a nyo ne nothing

d. Noone gave her anything

Wenn, wie Haegeman annimmt, in jedem der Sätze in (28) ein Negationskopf oberhalb der VP vorliegt und dessen Spec zumindest auf LF den Negationsausdruck oder, falls dieser als Quantor angehoben wurde, wenigstens dessen Spur beherbergt, ist es unklar, weshalb in (28c) der Polaritätsausdruck nicht lizenziert sein sollte. So wie in (28a, b, d) müsste ein stummer Neg-Kopf vorhanden sein, der das Auftreten von any legitimiert. Der Kontrast zwischen (28b) und (28c) ist dabei entscheidend: Wenn in (28b) der stumme Neg-Kopf, der sich vor der VP befinden müsste, die Lizenzierung von any leistet, dann muss er es auch in (28c) vermögen, unabhängig von der s-strukturellen Position des auf LF in die Spec-Position anzuhebenden NEG-Operators. Offensichtlich spielt aber nicht die stumme Kopf-Position sondern die s-strukturelle Position der negierten Indefinit-NP die entscheidende Rolle (vgl. Barss/Lasnik 1986, Haider 1993: 21). Sie muss den Polaritätsausdruck c-kommandieren. Anhebung der negierten NP auf LF, sei es nach Spec-Neg oder infolge von Quantorenanhebung an die Satzspitze liefert nicht die empirisch adäquaten Lizenzierungskontexte. Die Annahme eines stummen funktionalen Kopfes für die Negation ist daher nicht gerechtfertigt.

Wie unterscheidet sich die autonom-syntaktische Analyse von der LF-Analyse? Sie geht davon aus, dass bei der Konstruktion der semantischen Repräsentation der Abbildungsalgorithmus lediglich strukturelle syntaktische Verhältnisse zu berücksichtigen hat, also die Positionen auf der syntaktischen Struktur. Er bildet beispielsweise die c-KommandoVerhältnisse auf die Skopusverhältnisse ab. Daraus ergeben sich die Unterschiede in den Lesarten in (21), ebenso wie die Lizenzierungsunterschiede für Polaritätsausdrücke in 
(28). Ferner genügt es unter der autonom-syntaktischen Perspektive, zwischen Negation mittels Partikel und Negation mittels negierten Indefinitausdrucks zu unterscheiden:
a. weil niem and was gesehen hat
b. *weil nicht jemand/einer/Fritz was gesehen hat
c. *weil jemand nicht was gesehen hat
d. weil jemand was nicht gesehen hat

Die von der Negationspartikel c-kommandierte Domäne wird als negierte Domäne abgebildet. Eine negierte Indefit-NP ist semantisch ein negierter Quantor. Sein cKommandobereich ist der Bereich eines Quantors. Die negierten Indefinita werden direkt auf negierte Quantoren abgebildet und nicht als Ergebnis der Inkorporation von Negation in eine indefinite DP (,Negationsverschmelzung'). Die Negation mittels Partikel erzeugt hingegen ein negiertes Prädikat. Daraus erklärt sich der Kontrast zwischen Indefinitpronomina im Skopus eines negierten Quantors (29a) und Indefinitpronomina im Skopus einer Negationspartikel (29b, c). In der LF-Analyse würde in beiden Fällen unterschiedslos ein Negationskopf vorliegen. Dieser müsste allerdings dem Indefinitpronomen folgen. Daher müsste eine zusätzliche Umstellung angenommen werden, die die negierte Indefinit-NP vor das Objekt stellt. Auch diese erforderliche Umstellung entbehrt der Motivation.

Ein kurzer Vergleich mit der Fokuspartikel (s. auch Haider 1996) soll zeigen, dass es deutliche syntaktische Gemeinsamkeiten unter den Partikeln gibt. Daher ist die Nullhypothese die, dass die Negationspartikel nicht, syntaktisch betrachtet, nichts anderes als eine Partikel ist. Semantisch ist sie allerdings befrachtet mit der Negationsfunktion. Daraus ergibt sich ein spezifisches Domänenerfordernis: Soll die Partikel semantisch als Satznegation interpretiert werden, muss sie das finite Verbum in seiner Domäne haben. Daraus erklärt sich aus, warum die Negationspartikel im komplexen Vorfeld keine Satznegation sondern nur Konstituentennegation leisten kann.
a. (?) [Nicht in diesem Bett geschlafen] hat Napoleon
b. [So einen Vorfall zu melden] bräuchte man nicht
c. *[So einen Vorfall nicht zu melden] bräuchte man

(30a) ist zwar als Konstituentennegation (,nicht in diesem Bett, sondern ...') in Ordnung, als Satznegation aber nicht. Die Devianz von (30c) wird durch das Polaritätselement brauchen ausgelöst. Es verlangt Satznegation. Da es in (30c) nicht im Skopus der Negation ist, wenn diese Teil der Vorfeldkonstituente ist, führt dies zur Unakzeptabilität.

In VO-Sprachen tritt die Satznegation stets v or der VP auf. Das ergibt sich zwangsläufig aus dem Domänenerfordernis. Nur in der Position vor der VP hat die Negationspartikel die Grundposition des finiten Verbs, und damit auch seine Spur im Falle einer Verbvoranstellung, in seiner Domäne. In VO-Sprachen hingegen, kann die Negation dichter am Verb stehen (s. Dryer 1992: 97f für den typologischen Nachweis), da sie auch in VP-interner Position stets das Verb in seiner Domäne hat, denn das Verb folgt 
ja hinterdrein.

Betrachten wir nun Parallelen in der Distribution von Negationspartikelund Fokuspartikel. Beides sind Elemente, die, semantisch gesehen, über Mengen von Propositionen operieren. Negation bildet einen Satz auf die Komplementmenge der Erfüllungskontexte ab. Fokussierung ist ebenfalls eine Operation über Alternativenmengen (s. Rooth 1996).

Hier sind nun einige Gemeinsamkeiten in der Distribution von Negations- und Fokuspartikel:

i) Die Partikel folgt den vorangestellten Pronomina (31a, c).

ii) Die Partikel kann nicht in linksköpfigen Konstituenten auftreten $(32 \mathrm{a}, \mathrm{c})^{8}$

iii) Die Partikel kann mit einem extraponiertem Element a s s o zi i e rt sein (33)

31) a. *wenn nicht/nur sich jemand darum kümmerte

b. wenn sich nicht/nur jemand darum kümmerte

c. *wenn nicht/nur ihm jemand auf die Spur käme

d. wenn ihm nicht/nur jemand auf die Spur käme

32) a. *[Mit nur seiner Frau $]$ hat er gesprochen

b. [Nur mit seiner Frau] hat er gesprochen

c. *[Mit nicht seiner Frau] hat er gesprochen

d. [Nicht mit seiner Frau] hat er gesprochen

33) a. Er hat sie nicht angerufen, weil er sich geLANGweilt hat

b. Er hat sie nur angerufen, weil er sich geLANGweilt hat

c. Nicht weil er sich gelangweilt hat, hat er sie angerufen (sondern ...)

d. Nur weil er sich gelangweilt hat, hat er sie angerufen (sondern ...)

Die hier relevante Lesart für (33a, b) ist jene, die in (33c, d) paraphrasiert ist. In dieser Lesart bezieht sich die Negation in (33a) nur auf den extraponierten Satz und bildet keine Satznegation. In beiden Fällen muss die Partikel im Mittelfeld stehen und kann nicht zum extrapolierten Satz ins Nachfeld treten (s. Bayer 1991).

Das folgende Beispiel kombiniert rekursiv Anwendungen von Fokusierung und Negation.

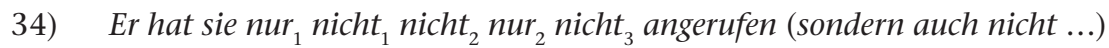

Die Partikel mit der jeweils größten Domäne sind propositionale Operatoren insofern sie als Domäne den gesamten Satz haben: Nur ${ }_{1}$ fokussiert die Proposition (Es ist nur so, dass ...). Nicht $t_{1}$ ist die Satznegation. Nicht $t_{2}$ und nicht ${ }_{3}$ sind Konstituentennegationen. Die Domäne von nur $_{2}$ ist die Konstituente nicht angerufen.

Wenden wir uns nun kurz den negativen Indefinitausdrücken zu. Sie werden gemäß der Neg-P-Hypothese als Spezifikator-Elemente eines stummen Negationskopfes gedeutet. 
Daraus erkläre sich die Funktion der Satznegation, wie z.B. in (35).

\section{5) Er hätte nichts zu sagen brauchen}

Die Satznegation, so die Annahme, werde durch den abstrakten Negationskopf geleistet, dessen Spezifikator den negativen Indefinitausdruck beherbergt. Der Indefinitausdruck steht in einer Kongruenzrelation (negative concord) zum Negationskopf, und ist nicht selbst der Negator. (36) jedoch zeigt, daß es keinen Platz für den abstrakten Negationskopf gibt. Das indefinite Objekt kann weder vor noch hinter der Negation stehen, wie in (36b, c) illustriert ist. Außerdem geht die Generalisierung verloren, daß ein Indefinitum nicht im Skopus der Negationspartikel stehen darf, wohl aber im Skopus eines negativen Indefinitums, wie eben in (36a). Dieser Unterschied läßt sich erfassen, wenn das negative Indefinitum in (36a) als negierter Existenzquantor gedeutet wird.

36) a. Dass keiner eine Ahnung hatte

b. *Dass er eine Ahnung $n$ icht hatte

c. *Dass er nicht eine Ahnung hatte

Folgendes Phänomen ließe sich allerdings mit der Annahme eines abstrakten NegKopfes elegant lösen. (37a) erlaubt eine Lesart (37b), in der die Negation Skopus über das Modal hat. Diese lässt sich nicht direkt aus der Interpretation des negierten Indefinitums als negierte Existenzquantifikation herleiten.

37) a. dass keine Lösungen bekannt sein müssen

b. Es muss nicht sein, dass es Lösungen gibt, die bekannt sind

Ähnliches gilt für das bereits von Bech (1955) konstatierte Datum, dass in kohärenten Konstruktionen ein Indefinitum die Satznegation für das Matrixverb leistet. So lässt sich etwa (38a) durch (38b) paraphrasieren.

\section{a. Er hat nichts zu beschönigen versucht \\ b. Er hat nicht versucht, etwas zu beschönigen}

Doch auch ohne Annahme eines Negationskopf lassen sich diese Phänomene erfassen, und zwar so, dass bei der Semantikkonstruktion das negierte Indefinitum nicht direkt in einen negierten Existenzoperator überführt wird, sondern als das interpretiert wird, was es sprachlich ist, nämlich als Kombination von Negation + Indefinitum. Diese Verknüpfung lässt sich semantisch als negierter Existenzquantor deuten, oder als Negationskennzeichnung einer Domäne auflösen. In vielen Sprachen, dazu gehören auch deutsche Dialekte, wird jedes Indefinitum in der Domäne als negativ markiert ('negative concord').

(39) I hob neambd nia nix gsogt (repräsentativ für bairisch-österreichische Dialekte)

'Ich habe niemandem nie nichts gesagt' (= Ich habe niemandem je etwas gesagt) 
Das Phänomen des 'negative concord' ist ein deutliches Indiz dafür, dass zwischen der morpho-syntaktischen Kennzeichnung und der semantischen Interpretation unterschieden werden muss. In (39) gibt es drei negierte Indefinita. Semantisch liegt aber eine einfache Satznegation vor. Die negierten Indefinita signalisieren die Negationsdomäne. Diese wird bei der Semantikkonstruktion als negiertes Prädikat gedeutet.

Zusammenfassend sei festgehalten: Die Syntax der Negation mittels Negationspartikel lässt sich als Spezialfall der Partikelsyntax erfassen, in Kombination mit den semantischen Konstruktionsbedingungen. Es gibt keine zwingenden Gründe dafür, und Evidenz dagegen, anzunehmen, dass die Syntax eine spezielle, d.h. inhärent semantisch typisierte Kategorie der Negation in Form einer Neg-P vorzusehen habe.

\section{Argumentstruktur}

Das Lexikon ist die zweite Komponente der Grammatik mit einer Phonologie- und Semantikschnittstelle. Die Argumentstruktur lexikalischer Köpfe bildet die Schnittstelle zwischen der lexikalisch-konzeptuellen Struktur und der syntaktisch relevanten Argumentausstattung. Es fällt sofort auf, dass es keine 1-zu-1-Beziehung zwischen Argumentstellen und semantischen Funktor-Argument-Beziehungen gibt: Ein Verb kann mehr syntaktische als logische Argumente haben (40a), oder es gibt weniger Argumentstellen als logische Argumente (40b).

40) a. nieseln, hageln, ... ; sich kümmern, sich wundern, ... ;

b. zubeißen, zugreifen, zuschlagen, ...

Quasi-Argumente wie die Subjekte von Wetterverben oder das Objekt eines inhärent reflexiven Verbs sind Beispiele für Argumentstellen ohne konzeptuelle Anbindung. Ableitungen von transitiven Verben wie in (40b) zeigen, dass einem konzeptuell vorhandenen Partizipanten keine Argumentstelle entspricht. Wenn jemand zugreift, gibt es etwas, das man ergreifen könnte. Das Verb stellt aber keine Argumentstelle dafür zur Verfügung. Dieser Umstand allein reicht aus, um einzusehen, dass keine direkte Abbildung von konzeptuellen Relationen auf syntaktische Beziehungen besteht.

Es wäre aber verfehlt, anzunehmen, dass die konzeptuelle Organisation und die Argumentstruktur nicht in einer systematischen Beziehung stünden. Die Frage ist bloß, ob die Argumentstruktur der verlängerte Arm der konzeptuellen Struktur sei, oder ob die konzeptuelle Struktur die semantische Bestückung der syntaktisch bereitgestellten Argumentformate steuere.

Das Faktum, dass bei Vorliegen einer Agensrelation diese bei jedem Verb an der ranghöchsten Argumentstelle kodiert ist, zeigt schon, dass die lexikalisch-konzeptuelle und die lexikalisch-syntaktische Organisation in Zusammenhang stehen. Dafür genügt es aber, eine allgemeine Abbildungsbeziehung zwischen beiden Strukturformaten zu formulieren: Das konzeptuell übergeordnete Argument entspricht der ranghöchsten Argumentstelle. 
Interessanter, weil diffiziler, ist die Frage, ob unterschiedliche Formattypen semantisch determiniert sind, oder ob auch im Bereich der Argumentstruktur der Variationsraum strukturell differenziert ist, und der Eindruck der semantischen Steuerung lediglich dadurch zustande kommt, dass ein Strukturformat einheitlich semantisch genutzt wird. In diesem Fall ist aber zu erwarten, dass dann, wenn die Parallele zwischen Struktur und Inhalt systematisch durchbrochen wird, das Strukturformat Vorrang vor dem semantischen Format hat. Als Evidenz für letzteren Fall lässt sich die Unterscheidung von intransitiven (41c) und unakkusativischen Verben (41b, d) heranziehen. Im Deutschen korreliert diese Unterscheidung weitgehend ${ }^{10}$ mit der Auxiliarwahl zwischen haben und sein als Auxiliar für die Perfektbildung.

41) a. Man hat den Stein auf die Straße gerollt

b. Der Stein ist im Bachbett gerollt

c. Sie haben auf der Straße getanzt

d. Sie sind auf die Straße getanzt

Was die unakkusativischen Verben von den transitiven und intransitiven unterscheidet, ist das Fehlen des designierten Arguments (s. Haider 1984). Es ist jenes der beiden Argumente mit struktureller Kasuseigenschaft im Format des transitiven Verbs, das Vorrang für die Subjektfunktion hat. In $(42 \mathrm{a}, \mathrm{b})$ ist es durch Unterstreichung designiert.
a. $\mathrm{V}^{\circ} \quad<\underline{\mathrm{A}}, \mathrm{A}, \ldots>$
transitiv
b. $V^{\circ}$
$<\underline{A}, \ldots>$
intransitiv
c. $\mathrm{V}^{\circ} \quad<\mathrm{A}, \ldots>$
unakkusativisch

Transitive und intransitive Verben unterscheiden sich am nicht-designierten Argument. Ein intransitives Verb hat nur ein strukturelles Argument (neben allfällig zusätzlichen Argumenten mit inhärentem Kasus), und das ist das designierte. Unakkusativische Verben hingegen haben kein designiertes Argument. Ihr im Subjektskasus realisiertes Argument ist strukturell betrachtet das gleiche Argument, wie das Objektsargument eines transitiven Verbs. Nominativzuweisung maskiert diesen Unterschied, denn auch beim transitiven Verb erhält das Objekt den Subjektskasus, wenn der Subjektsaktant fehlt, wie im Falle von Passiv.

Subjekte unakkusativischer Verben sind Objektsaktanten und verhalten sich syntaktisch auch so. Dies lässt sich besonders gut bei Resultativ-Prädikation erkennen. Das resultative Prädikat kann sich nur auf direkte Objekte oder eben Subjekte von unakkusativischen Verben beziehen. In anderen Worten, es bezieht sich auf das nicht-designierte strukturelle Argument.
a. Er hetzte die Meute müde $^{\mathrm{i}}$
transitiv
b. Er lachte sich ${ }^{\mathrm{i}}$ krumm $^{\mathrm{i}}$
intransitiv
c. Er zerbrach in drei Stücke ${ }^{\mathrm{i}}$
unakkusativisch 
In (43a) kann sich müde als resultative Prädikation (n.b. nicht zu verwechseln mit der depiktiven Interpretation), also in der Interpretation, dass im Nachzustand der Handlung die Eigenschaft eintritt, nur auf das Objekt, nicht aber auf das Subjekt beziehen. Instruktiv ist (43b): Will man ein Resultativ auf ein designiertes Argument beziehen, muss man ein Reflexivum als Objekt dazwischenschalten. Dieses bezieht sich auf das Subjekt, und damit kann das Objektsprädikat indirekt auf den Subjektreferenten applizieren. (43c) schließlich zeigt, dass nur bei unakkusativischen Verben Subjektsbezug vorliegt. Bei der Untersuchung der unakkusativischen Verben könnte man zur Auffassung gelangen, dass die Semantik des Verbs ursächlich sei dafür, was ein intransitives und was ein unakkusativisches Verb ist. Unakkusativische Verben sind typischerweise telisch oder terminativ und ihr struktureller Aktant ist häufig in einer Thema-Relation. Doch diese Eigenschaften sind weder notwendig noch hinreichend. Das Verb in (41b) ist unakkusativisch, aber es ist nicht terminativ. Andererseits gibt es terminative Themaverben, die nicht unakkusativisch sind, wie z.B. abheben (vgl. Das Flugzeug hat abgehoben).

Dass die Semantik in ihren Rechten durch die strukturellen Bedingungen beschränkt ist, sieht man am deutlichsten, wenn man zwei Verben vergleicht, die nahezu synonym sind, aber formal (über die Auxiliarselektion) als intransitiv bzw. unakkusativisch ausgewiesen sind.

(44) a. Das Defizit ist (zu einer großen Belastung) angewachsen

b. Das Defizit hat ( ${ }^{*} z$ e einer großen Belastung) zugenommen

Anwachsen ist als Ableitung eines unakkusativischen Verbs selbst auch unakkusativisch, zunehmen ist die Ableitung eines transitiven Verbs und selbst intransitiv (vgl. 40b). Daraus folgt der Unterschied in der Bezugsmöglichkeit eines Resultativprädikats. Der semantischen Ähnlichkeit zum Trotz gehören die beiden Verben nicht in dieselbe Formklasse.

\section{SCHLUSSBEMERKUNG}

Anhand der Diskussion der drei Phänomenbereiche sollte gezeigt werden, dass die syntaktische und die semantische Strukturierung zwar - wie nicht anders möglich - aufeinander abgestimmt sind, dass sich aber die syntaktische Struktur nicht als bloß formaler, für die inhaltliche Befrachtung geeignet zurechtgelegter Unterbau der Semantik verstehen lässt. Die syntaktische Strukturierung folgt eigenen Prinzipien, die nicht reduzierbar sind auf semantische Primitiva.

\section{LITERATUR}

Barss, Andrew, Howard Lasnik (1986), "A Note on Anaphory and Double Objects", Linguistic Inquiry, 17 (1986), pp. 347-354.

Bayer, Josef (1991), Directionality of Government and Logical Form. A Study of Focusing Particles and Wh-Scope (Habilitationsschrift, Univ. Konstanz). 
Bech, Gunnar(1955/1957), Studienüberdas deutsche Verbuminfinitum, 2 Bde., Kopenhagen: Munksgaard (2. Aufl. 1983 von C. Fabricius-Hansen, Tübingen: Niemeyer).

Chomsky, Noam (1995), "Categories and Transformations", in: Chomsky, Noam (1995), The Minimalist Program, Cambridge/Mass.: MIT Press, pp. 219-394.

Cinque, Guglielmo (1999), Adverbs and Functional Heads: a Cross-Linguistic Perspective, Oxford: Oxford University Press.

Davidson, Donald (1980), Essays on Actions and Events, Oxford: Clarendon Press.

Dryer, Mathew S. (1992), "The Greenbergian word order correlations", Language, 68 (1992), pp. 81-138.

Ernst, Thomas (2002), The Syntax of Adjuncts, Cambridge: Cambridge University Press.

Haegeman, Liliane (1995), The Syntax of Negation, Cambridge: Cambridge University Press.

Haider, Hubert (1984), "The Case of German", in: Toman, J. (ed.) (1984), Studies in German Grammar, Dordrecht: Foris, pp. 65-102.

Haider, Hubert (1993), Deutsche Syntax, generativ. Vorstudien zur Theorie einer projektiven Grammatik, Tübingen: Narr.

Haider, Hubert (1996), "Wenn die Semantik arbeitet - und die Syntax sie gewähren läßt", in: Harras, Gisela, Manfred Bierwisch (Hgg.) (1996), Wenn die Semantik arbeitet. Festschrift für Klaus Baumgärtner, Tübingen: Niemeyer, pp. 7-27.

Haider, Hubert (2005), "How to turn German into Icelandic", The Journal of Comparative Germanic Linguistics, 8 (2005), pp. 1-53.

Hornstein, Norbert (1995), Logical Form. From GB to Minimalism, Oxford: Blackwell.

Jacobs, Joachim (1991), "Negation", in: Stechow, Arnim von, Dieter Wunderlich (Hgg.) (1991), Semantik. Ein internationales Handbuch der zeitgenössischen Forschung, Berlin: Mouton de Gruyter, pp. 560-596.

Kamp, Hans \& Uwe Reyle. 1993. From Discourse to Logic I. Dordrecht: Reidel.

Pollock, Jean-Yves (1989), "Verb Movement, Universal Grammar, and the Structure of IP", Linguistic Inquiry, 20 (1989), pp. 365-424.

Rooth, Mats (1996), "Focus", in: Lappin, S. (ed.) (1996), The Handbook of Contemporary Semantic Theory, Berlin: de Gruyter, pp. 271-297.

\section{ANMERKUNGEN}

1 Eine VO Sprache ist eine Sprache, in der das Kopfelement (im Falle der VP also das Verb) den von ihm abhängigen Elementen vorausgeht. In einer OV Sprache geht das Kopfelement hinterdrein.

2 Damit scheidet eine Analyse aus, die sekundäre Pradikate wie in (10c) als Prädikate eines, small clause' auffaßt. Läge in (10c) ein small clause vor, gäbe es auch eine Prädikation, auf die sich das Satzadverb beziehen könnte. Das folgende Beispiel zeigt, dass Satzadverbien bei small clauses möglich sind: Er ist [leider völlig unzufrieden mit dem Verhandlungsresultat] vorzeitig abgereist.

3 Kopf-initiale Phrasen sind kompakt, insofern zwischen den Kopf und die nachfolgenden Argumente keine Adverbien dazwischentreten können. Dies gilt für kopf-finale Strukturen nicht. Dies ist eine ausschließlich syntaktisch bedingte Eigenschaft (s. Haider 2005).

4 Eine Partikel ist ein Element ohne kategoriale Spezifikation, das potentiell an jede Konstituente adjungiert werden kann. 
5 Scrambling ergibt eine Voranstellung von Elementen im Mittelfeld.

6 Jacobs (1991: 573) formuliert das so: Wenn Negationsträger als Nicht-Köpfe realisiert werden (etwa als Adjunkte), sollten sie eine Position haben, von der aus das Prädikat in ihrem syntaktischen Bereich liegt.

7 Prädikative APs gehen mit der Kopula eine kohärente Konstruktion ein, das heißt, die Köpfe bilden einen komplexen Kopf und das Komplement des abhängigen Elements wird zum Komplement des komplexen Kopfes (s. Haider 1993, Kap. 9.6).

8 Die Beschränkung gilt nicht bloß für die PP-interne Positionierung. Gleiches gilt für NPs ( ${ }^{*}$ die Nachrichten nur/nicht von Fritz). APs hingegen, da rechtsköpfig, sind zulässig (der nur in Österreich bekannte Vorfall).

9 Den Hinweis darauf verdanke ich Angelika Kratzer, die die urspüngliche Beobachtung Arnim von Stechow zuschreibt.

10 Es gibt einige transitive sein-Verben. Diese sind aber alle von einem unakusativischen Verb abgeleitet, wie z.B.: den Ablauf nochmals durchgehen, den Ehebund eingehen; den Bedürfnissen nachkommen; ... 